\title{
Semiparametric Estimation of Multivariate GARCH Models
}

\author{
Claudio Morana $1,2,3$ \\ ${ }^{1}$ Department of Economics, Management and Statistics, University of Milan-Bicocca, Milan, Italy \\ ${ }^{2}$ Center for Research on Pensions and Welfare Policies, Collegio Carlo Alberto, Moncalieri, Italy \\ ${ }^{3}$ Rimini Center for Economic Analysis, Rimini, Italy \\ Email: Claudio.Morana@unimib.it
}

Received 30 November 2015; accepted 27 December 2015; published 30 December 2015

Copyright (C) 2015 by author and Scientific Research Publishing Inc.

This work is licensed under the Creative Commons Attribution-NonCommercial International License (CC BY-NC). http://creativecommons.org/licenses/by-nc/4.0/

(c) (1) \&) Open Access

\begin{abstract}
The paper introduces a new simple semiparametric estimator of the conditional variance-covariance and correlation matrix (SP-DCC). While sharing a similar sequential approach to existing dynamic conditional correlation (DCC) methods, SP-DCC has the advantage of not requiring the direct parameterization of the conditional covariance or correlation processes, therefore also avoiding any assumption on their long-run target. In the proposed framework, conditional variances are estimated by univariate GARCH models, for actual and suitably transformed series, in the first step; the latter are then nonlinearly combined in the second step, according to basic properties of the covariance and correlation operator, to yield nonparametric estimates of the various conditional covariances and correlations. Moreover, in contrast to available DCC methods, SP-DCC allows for straightforward estimation also for the non-symultaneous case, i.e. for the estimation of conditional cross-covariances and correlations, displaced at any time horizon of interest. A simple ex-post procedure to ensure well behaved conditional variance-covariance and correlation matrices, grounded on nonlinear shrinkage, is finally proposed. Due to its sequential implementation and scant computational burden, SP-DCC is very simple to apply and suitable for the modeling of vast sets of conditionally heteroskedastic time series.
\end{abstract}

\section{Keywords}

Multivariate GARCH Model, Dynamic Conditional Correlation, Semiparametric Estimation

\section{Introduction}

Since the seminal contribution of [1], the literature on multivariate Generalized Autoregressive Conditional He- 
teroskedasticity (GARCH) models has rapidly developed (see [2] and [3], for surveys). To date, three generations of models can be counted. First generation models, likewise the VEC model of [1] and the BEKK model of [4], are straightforward extensions of the univariate GARCH model. They allow for very general conditional variance covariance matrix dynamics, yet at the cost of a very profligate parameterization, which limits their use to small sets of time series. This drawback has been overcome by second generation models, yet at the cost of imposing either parameter restrictions on the BEKK model, as for the case of the scalar BEKK model and the exponentially weighted moving average model introduced by [5], or on the conditional correlation matrix, assumed time-invariant in the constant conditional correlation CCC model of [6]. Alternatively, restrictions have been imposed through factor structures, likewise [7] and the orthogonal models of [8]-[10] and [11]. On the other hand, a different approach has been pursued by the most recent third generation of multivariate GARCH models, i.e. the dynamic conditional correlation models, grounded on a two-step estimation procedure, involving the estimation of univariate GARCH models for the conditional variances in the first step and then the estimation of the conditional covariances in the second step. Although inefficient, the latter sequential procedure is consistent and asymptotically normal. Moreover, by dramatically reducing the numerical optimization burden, it can be implemented also in the case of vast sets of time series. In this respect, seminal is the Dynamic Conditional Correlation models (DCC) of [12] and [13]. Further extensions are [14], the Dynamic Conditional EquiCorrelation (DECO) model of [15], [16] and [17].

Dynamic conditional correlation models, in order to ensure positive definiteness of the conditional variance-covariance matrix, posit the correlation matrix to be a transformation of a latent matrix, which is a function of past devolatilized innovations. In particular, while the CCC model of [6] assumes time invariant, but pairwise specific correlations, the DECO model of [15] makes the opposite assumption, positing time varying correlations, but equal across series. Both CCC and DECO therefore rely on assumptions on conditional correlation dynamics which are unlikely to be supported by the data. On the other hand, in the alternative formulation of [13], the correlation matrix is modeled directly and as a function of past correlations of devolatilized innovations. As a common drawback, all of the available dynamic conditional correlation models rely on the choice, neither unique nor obvious, of a long run target for the conditional variance-covariance or correlation matrix.

In the light of the above issues, the paper then contributes to the literature by introducing a new simple semiparametric estimator of the conditional variance-covariance and correlation matrix (SP-DCC). While sharing a similar sequential approach to DCC and DECO, SP-DCC has the advantage of not requiring the direct parameterization of the conditional covariance or correlation processes, therefore also avoiding any assumption on their long-run target. In the proposed framework, conditional variances are estimated by univariate GARCH models, for actual and suitable transformed series, in the first step; the latter are then nonlinearly combined in the second step, according to basic properties of the covariance and correlation operator, to yield nonparametric estimates of the corresponding conditional covariances and correlations. In contrast to available DCC methods, SP-DCC allows for straightforward estimation also for the non-symultaneous case, i.e. for the estimation of conditional cross-covariances and correlations displaced at any time horizon of interest. A simple ex-post procedure to ensure well behaved conditional covariance and correlation matrices, grounded on nonlinear shrinkage, is finally proposed. Due to its sequential implementation and scant computational burden, SP-DCC is very simple to apply and suitable for the modeling of vast sets of conditionally heteroskedastic time series. We point to [18] for an empirical application of the proposed approach.

\section{Semiparametric Estimation of Dynamic Conditional Correlations}

Consider a discrete time, real-valued vector stochastic process $\left\{y_{t}\right\}$ of dimension $N \times 1$

$$
y_{t}=\mu_{t}(\theta)+\varepsilon_{t}
$$

where $\mu_{t}(\theta)$ is the conditional mean vector $E\left(y_{t} \mid I_{t-1}\right), \theta$ is a vector of parameter, $I_{t-1}$ is the sigma field, and

$$
\varepsilon_{t}=H_{t}^{1 / 2}(\theta) z_{t}
$$

where $H_{t}^{1 / 2}(\theta)$ is a positive definite matrix of dimension $N \times N$.

The random vector $z_{t}$ is of dimension $N \times 1$ and assumed to be i.i.d. with first two moments 


$$
\begin{gathered}
E\left(z_{t}\right)=0 \\
\operatorname{Var}\left(z_{t}\right)=I_{N}
\end{gathered}
$$

where $I_{N}$ is the identity matrix of dimension $N$.

It is straightforward to show that $H_{t}(\theta)$ is the conditional variance-covariance matrix; in fact

$$
\begin{aligned}
\operatorname{Var}\left(y_{t} \mid I_{t-1}\right) & \equiv \operatorname{Var}_{t-1}\left(y_{t}\right)=\operatorname{Var}_{t-1}\left(\varepsilon_{t}\right) \\
& =H_{t}^{1 / 2}(\theta) \operatorname{Var}_{t-1}\left(z_{t}\right) H_{t}^{1 / 2}(\theta)^{\prime} \\
& =H_{t}(\theta) .
\end{aligned}
$$

In general both $\mu_{t}$ and $H_{t}$ depend on the parameter vector $\theta$. While, the conditional mean vector does not depend on the conditional variance parameter, apart from the GARCH-in-mean case, the conditional variance matrix depends on the conditional mean parameters through the residuals. In what follow, for simplicity, we leave out $\theta$ from notation and neglect the conditional mean vector, which might be modelled in various ways, i.e. by means of univariate or multivariate ARMA models.

\subsection{The Conditional Variance Process}

We assume the elements along the main diagonal of $H_{t}$ follow a GARCH $(1,1)$ process

$$
h_{i i, t}=\omega_{i}+\alpha_{i} \varepsilon_{i, t-1}^{2}+\beta_{i} h_{i, t-1} \quad i=1, \cdots, N
$$

subject to the usual restrictions required to ensure that the generic ith conditional variance process

$\operatorname{Var}_{t-1}\left(y_{i, t}\right) \equiv h_{i i, t}$ is positive almost surely at any point in time. For instance, sufficient (not necessary) conditions are $\omega_{i}>0, \alpha_{i} \geq 0, \beta_{i} \geq 0$, with stationarity condition $\alpha_{i}+\beta_{i}<1 .{ }^{1}$

An extended specification is in principle also viable, i.e.

$$
h_{i i, t}=\omega_{i}+\alpha_{i, 1} \varepsilon_{1, t-1}^{2}+\cdots+\alpha_{i, N} \varepsilon_{N, t-1}^{2}+\beta_{i, 1} h_{1, t-1}+\cdots+\beta_{i, N} h_{N, t-1} \quad i=1, \cdots, N
$$

yet actually feasible only for small $N$.

\subsection{The Conditional Covariance Process}

Consider the identity

$$
\operatorname{Cov}(A, B) \equiv \frac{1}{4}[\operatorname{Var}(A+B)-\operatorname{Var}(A-B)]
$$

given that $\operatorname{Var}(A \pm B)=\operatorname{Var}(A)+\operatorname{Var}(B) \pm 2 \operatorname{Cov}(A, B)$.

The off-diagonal elements of $H_{t}$ can then be defined accordingly, i.e.

$$
\operatorname{Cov}_{t-1}\left(y_{i, t}, y_{j, t}\right) \equiv h_{i j, t}=\frac{1}{4}\left[\operatorname{Var}_{t-1}\left(y_{i, t}+y_{j, t}\right)-\operatorname{Var}_{t-1}\left(y_{i, t}-y_{j, t}\right)\right] \quad i, j=1, \cdots, N \quad i \neq j .
$$

By defining the new variables $y_{i j, t}^{+} \equiv y_{i, t}+y_{j, t}$ and $y_{i j, t}^{-} \equiv y_{i, t}-y_{j, t}$, and assuming a GARCH $(1,1)$ specification for their conditional variance processes $\operatorname{Var}_{t-1}\left(y_{i j, t}^{+}\right) \equiv h_{i j, t}^{+}$and $\operatorname{Var}_{t-1}\left(y_{i j, t}^{-}\right) \equiv h_{i j, t}^{-}$

$$
\begin{aligned}
& \operatorname{Var}_{t-1}\left(y_{i j, t}^{+}\right) \equiv h_{i, t}^{+}=\omega_{i, j}^{+}+\alpha_{i, j}^{+} \varepsilon_{i, j, t-1}^{+2}+\beta_{i, j}^{+} h_{i, t-1}^{+} \quad i, j=1, \cdots, N \quad i \neq j \\
& \operatorname{Var}_{t-1}\left(y_{i j, t}^{-}\right) \equiv h_{i j, t}^{-}=\omega_{i, j}^{-}+\alpha_{i, j}^{-} \varepsilon_{i, j, t-1}^{-2}+\beta_{i, j}^{-} h_{i, t-1}^{-} \quad i, j=1, \cdots, N \quad i \neq j
\end{aligned}
$$

subject to the usual restrictions required to ensure a well behaved conditional variance process, (7) becomes

$$
h_{i j, t}=\frac{1}{4}\left[h_{i j, t}^{+}-h_{i j, t}^{-}\right] \quad i, j=1, \cdots, N \quad i \neq j .
$$

${ }^{1}$ The GARCH $(1,1)$ model is chosen for simplicity; the approach is very flexible and can accommodate any model of the GARCH family. 
Moreover, if residuals are obtained from linear transformations of the original variables ${ }^{2}$, then $\varepsilon_{i j, t-1}^{+2}=\left(\varepsilon_{i, t-1}+\varepsilon_{j, t-1}\right)^{2}$ and $\varepsilon_{i j, t-1}^{-2}=\left(\varepsilon_{i, t-1}-\varepsilon_{j, t-1}\right)^{2}$; hence, (8) and (9) can be written as

$$
\begin{aligned}
& h_{i j, t}^{+}=\omega_{i, j}^{+}+\alpha_{i, j}^{+}\left(\varepsilon_{i, t-1}+\varepsilon_{j, t-1}\right)^{2}+\beta_{i, j}^{+} h_{i, t-1}^{+} \quad i, j=1, \cdots, N \quad i \neq j \\
& h_{i j, t}^{-}=\omega_{i, j}^{-}+\alpha_{i, j}^{-}\left(\varepsilon_{i, t-1}-\varepsilon_{j, t-1}\right)^{2}+\beta_{i, j}^{-} h_{i, t-1}^{-} \quad i, j=1, \cdots, N \quad i \neq j .
\end{aligned}
$$

By means of the proposed method conditional cross-covariances and correlations can also be computed, as

$$
\operatorname{Cov}_{t-1}\left(y_{i, t}, y_{j, t-k}\right)=\frac{1}{4}\left[\operatorname{Var}_{t-1}\left(y_{i, t}+y_{j, t-k}\right)-\operatorname{Var}_{t-1}\left(y_{i, t}-y_{j, t-k}\right)\right] \quad i, j=1, \cdots, N \quad i \neq j, k \neq 0 .
$$

\subsection{Estimation}

Consistent and asymptotically normal estimation is performed in two steps.

Firstly, the conditional variances $h_{i, t}, i=1, \cdots, N$, i.e. the elements along the main diagonal of $H_{t}$, and $h_{i j, t}^{+}$, $h_{i j, t}^{-}, i, j=1, \cdots, N, i \neq j$, are estimated equation by equation by means of $Q M L$; this yields $\hat{h}_{i, t}$, $i=1, \cdots, N$, and $\hat{h}_{i j, t}^{+}, \hat{h}_{i j, t}^{-}, \quad i, j=1, \cdots, N, \quad i \neq j$.

Then, in the second step the off-diagonal elements of $H_{t}, h_{i j, t}, i, j=1, \cdots, N, i \neq j$, are estimated nonparametrically by computing

$$
\hat{h}_{i j, t}=\frac{1}{4}\left[\hat{h}_{i j, t}^{+}-\hat{h}_{i j, t}^{-}\right] \quad i, j=1, \cdots, N \quad i \neq j .
$$

By defining

$$
\hat{D}_{t}=\operatorname{diag}\left(\hat{h}_{1, t}^{1 / 2}, \cdots, \hat{h}_{N, t}^{1 / 2}\right)
$$

the conditional correlation matrix $R_{t}$ is then estimated as

$$
\hat{R}_{t}=\hat{D}_{t}^{-1} \hat{H}_{t} \hat{D}_{t}^{-1} .
$$

By definition the matrix $H_{t}$ is positive definite; our estimation approach does not restrict $\hat{H}_{t}$ to be almost surely positive definite at any point in time. The latter property can however be checked ex-post by computing the eigenvalues of $\hat{H}_{t}$, which by being a real, square and symmetric matrix, under positive definiteness are expected to be all positive. In practice this can be performed by means of Descartes' rule of alternating signs applied to its characteristic polynomial ${ }^{3}$, as well as by means of Sylvester's criterion ${ }^{4}$, or by assessing the existence and uniqueness of its Cholesky decomposition.

However, the positive definiteness property might also be imposed ex-post, by means of shrinkage methods, as in Ledoit and Wolf $(2004,2012)$. In the latter case a compromise estimate of the conditional correlation matrix $\hat{R}_{t}^{c}$ is obtained by shrinking the estimated conditional correlation matrix $\hat{R}_{t}$ towards the identity matrix, i.e. by computing

$$
\hat{R}_{t}^{c}=\lambda_{t} I_{N}+\left(1-\lambda_{t}\right) \hat{R}_{t}
$$

where $\lambda_{t}$ is the shrinkage intensity at time period $t$. The compromise estimate of $H_{t}$, i.e., $\hat{H}_{t}^{c}$ can then be

\footnotetext{
${ }^{2}$ This, for instance, would occur when the conditional mean vector is specified as a vector autoregressive (VAR) process, yet not in the presence of a VARMA structure. In the latter case residuals $\varepsilon_{i, j, t}^{+}$and $\varepsilon_{i, j, t}^{-}$should be computed from time series models specified for the new variables $y_{i, t}^{+}$and $y_{i, t}^{-}$.

${ }^{3} \mathrm{By}$ ordering the terms of the characteristic polynomial with real coefficients by descending variable exponent, the number of positive roots of the polynomial is then either equal to the number of sign differences between consecutive nonzero coefficients, or is less than it by an even number. Multiple roots of the same value are counted separately.

${ }^{4}$ According to Sylvester's criterion, a real, square symmetric positive definite matrix shows all positive leading principal minors, where the $k$ th leading principal minor of a matrix $M$ is the determinant of its upper-left $k$ by $k$ sub-matrix. In practice the $M$ matrix is reduced to an upper triangular matrix by means of row operations, as in the first part of the Gaussian elimination method, preserving the sign of its determinant during pivoting process. Since the $k$ th leading principal minor of a triangular matrix is the product of its diagonal elements up to row $k$, positive definiteness can be assessed by checking whether its diagonal elements are all positive. The latter condition is then checked each time a new row $k$ of the triangular matrix is obtained.
} 
obtained as

$$
\hat{H}_{t}^{c}=\hat{D}_{t} \hat{R}_{t}^{c} \hat{D}_{t}
$$

which is positive definite by construction, as $\hat{R}_{t}^{c}$ is positive definite and the elements of $\hat{D}_{t}$ are well-defined.

\subsection{Ex-Post Correction for Well-Behaved Conditional Covariances and Correlations}

Alternatively, the validity of the Cauchy-Schwarz inequality and the condition of positive definiteness can be imposed sequentially, at each point in time $t$, following the below procedure.

Firstly, the estimated conditional correlations in $\hat{R}_{t}, \hat{\rho}_{i j}, i, j=1, \cdots, N, i \neq j$, are bounded to lie within the range $-1 \leq \hat{\rho}_{i j} \leq 1$, by applying the sign-preserving bounding transformation

$$
\hat{\rho}_{i j}^{*}=\hat{\rho}_{i j}\left(1+\hat{\rho}_{i j}^{k}\right)^{-1 / k}
$$

where $k>0$ and even; the value of $k$ can be selected optimally by solving

$$
\min _{k} \sum_{t=1}^{T}\left\|\hat{R}_{t}-\hat{R}_{t}^{*}\right\|_{F}=\min _{k} \sum_{t=1}^{T} \sqrt{\sum_{i=1}^{N} \sum_{j=1}^{N}\left|\hat{\rho}_{i j, t}-\hat{\rho}_{i j, t}^{*}\right|^{2}}
$$

i.e. by setting $k$ in such a way that the sum of Frobenious norms over the temporal sample is minimized; this yields $\hat{R}_{t}^{*}$, the transformed correlation matrix, which satisfies, by construction, the Cauchy-Schwarz inequality.

Secondly, positive definiteness is enforced by computing the eigenvalue-eigenvector decomposition of the transformed conditional correlation matrix $\hat{R}_{t}^{*}$, yielding

$$
\hat{R}_{t}^{*}=\hat{E}_{t} \hat{V}_{t} \hat{E}_{t}^{\prime}
$$

where $\hat{V}_{t}$ is the $N \times N$ diagonal matrix containing the $N$ ordered eigenvalues along the main diagonal, and $\hat{E}_{t}$ is the $N \times N$ matrix containing the $N$ associated orthogonal eigenvectors. In the case of violation of the positive definiteness condition one or more of the eigenvalues will be negative; an empirically viable strategy to impose positive definiteness ex-post consists of replacing the negative sample eigenvalues with positive values, computed for instance from their sample average value when positive or from the grand average across sample eigenvalues. The rationale guiding this practice is the well-known issue of downward biased estimation of the smallest eigenvalues (versus upward biased estimation of the largest eigenvalues). Rather than shrinking all the sample eigenvalues towards their grand average, as occurring by implementing [19], only the negative eigenvalues are shrank towards positive average values. The latter practice is consistent with nonlinear shrinkage of the covariance matrix ([20]), allowing in principle for different shrinkage intensities to be applied to the various eigenvalues.

The shrank matrix of eigenvalues $\hat{V}_{t}^{*}$ would then be obtained, and therefore

$$
\hat{R}_{t}^{* *}=\hat{E}_{t} \hat{V}_{t}^{*} \hat{E}_{t}^{\prime}
$$

which, by construction, is well-behaved at each point in time. The implied conditional covariance process at time period $t$ can then be obtained as

$$
\hat{H}_{t}^{* *}=\hat{D}_{t} \hat{R}_{t}^{* *} \hat{D}_{t}
$$

where

$$
\hat{D}_{t}=\operatorname{diag}\left(\hat{h}_{1, t}^{1 / 2}, \cdots, \hat{h}_{N, t}^{1 / 2}\right)
$$

as before. The implied estimated variance-covariance matrix $\hat{H}_{t}^{* *}$ then obeys the Cauchy-Schwarz inequality and the positive definiteness condition, at each point in time, by construction.

\subsection{Asymptotic Properties}

Under assumptions (1) through (5), estimation and inference for the parameters of the univariate GARCH $(1,1)$ processes (5), (8) and (9) can be performed by means of $Q M L$. The Gaussian log likelihood function for the generic process $y_{i, t}$, assuming for simplicity $E\left(y_{i, t} \mid I_{t-1}\right)=0$ and a GARCH $(1,1)$ structure 
$h_{i, t}=\omega_{i}+\alpha_{i} y_{i, t-1}^{2}+\beta h_{i, t-1}$ can then be written as

$$
\ln \left\{L\left(\vartheta, y_{i, t}, \cdots, y_{i, T}\right)\right\}=-0.5 T \ln (2 \pi)-0.5 \sum_{t=1}^{T}\left\{\ln \left(h_{i, t}\right)+y_{i, t}^{2} h_{i, t}^{-1}\right\}
$$

and numerically maximized with respect to the vector of parameters $\vartheta=\left(\omega_{i}, \alpha_{i}, \beta_{i}\right)^{\prime}$. Similarly for the other variables $y_{i, j, t}^{+}$and $y_{i, j, t}^{-}$.

Under fairly general conditions, the asymptotic distribution of $Q M L$ is

$$
T^{1 / 2}\left(\hat{\vartheta}-\vartheta_{0}\right) \rightarrow N\left\{\mathbf{0}, \mathbf{A}\left(\boldsymbol{\vartheta}_{0}\right)^{-1} \mathbf{B}\left(\boldsymbol{\vartheta}_{0}\right) \mathbf{A}\left(\boldsymbol{\vartheta}_{0}\right)^{-1}\right\}
$$

where $\vartheta_{0}$ denotes the true value of the vector of parameters, and where $\mathbf{A}\left(\vartheta_{0}\right)$ is the Hessian and $\mathbf{B}\left(\boldsymbol{\vartheta}_{0}\right)$ is the outer product gradient, both of which are evaluated at the true parameter values. This also establishes the consistent and asymptotically normal estimation of the conditional variance of $y_{t}, E\left(y_{i, t}^{2} \mid I_{t-1}\right)$, as well as of the transformed variables $y_{i j, t}^{+}$and $y_{i j, t}^{-}$.

Consistent and asymptotically normal estimation of the off-diagonal elements of the conditional variance-covariance matrix $H_{t}$ then follows directly from the consistent and asymptotically normal estimation of the conditional variances of the transformed variables in (8) and (9). In fact, considering the generic off-diagonal $i, j$ element of $H_{t}, i, j=1, \cdots, N, i \neq j$, one has

$$
\widehat{\operatorname{Cov}}_{t-1}\left(y_{i, t}, y_{j, t}\right)=\frac{1}{4}\left[\widehat{\operatorname{Var}}_{t-1}\left(y_{i, t}+y_{j, t}\right)-\widehat{\operatorname{Var}}_{t-1}\left(y_{i, t}-y_{j, t}\right)\right]
$$

as the conditional covariance estimator $\overparen{\operatorname{Cov}}_{t-1}\left(y_{i, t}, y_{j, t}\right)$ is a linear combination of the (consitent and asymptotically normal) conditional variance estimators for the transformed variables $\widetilde{\operatorname{Var}}_{t-1}\left(y_{i, t}+y_{j, t}\right)$ and $\widehat{\operatorname{Var}}_{t-1}\left(y_{i, t}-y_{j, t}\right)$.

\section{Conclusion}

The paper introduces a new simple semiparametric estimator of the conditional variance-covariance and correlation matrix (SP-DCC). While sharing a similar sequential approach to existing dynamic conditional correlation methods, SP-DCC has the advantage of not requiring the direct parameterization of the conditional covariance or correlation processes. In the first step, conditional variances are estimated by univariate GARCH models for actual and suitably transformed series. In the second step, the estimated conditional covariances are then nonlinearly combined, according to basic properties of the covariance and correlation operator, to yield nonparametric estimates of the various conditional covariances and correlations. At this step, SP-DCC also allows for the estimation of conditional cross-covariances and correlations, displaced at any time horizon. In the third step, well behaved conditional variance-covariance and correlation matrices are obtained by means of nonlinear shrinkage. Due to its sequential implementation and scant computational burden, SP-DCC is very simple to apply and suitable for the modeling of vast sets of conditionally heteroskedastic time series.

\section{Acknowledgements}

The author is grateful to the referee, M. Rockinger and M. Dacorogna for their comments. This project has received funding from the European Union's Seventh Framework Programme for research, technological development and demonstration under grant agreement no. 3202782013-2015. On rainy days, be in the rain/In windy days, be in the wind (Mitsuo Aida).

\section{References}

[1] Bollerslev, T., Engle, R.F. and Wooldridge, J. (1988) A Capital Asset Pricing Model with Time Varying Covariances. Journal of Political Economy, 96, 116-131. http://dx.doi.org/10.1086/261527

[2] Bauwens, L., Laurent, S. and Rombouts, L. (2006) Multivariate GARCH Models: A Survey. Journal of Applied Econometrics, 21, 79-109. http://dx.doi.org/10.1002/jae.842

[3] Silvennoinen, A. and Terasvirta, T. (2009) Multivariate GARCH Models. In: Andersen, T., Davis, R., Kreiss, J.F. and Mikosch, T., Eds., Handbook of Financial Time Series, Springer, Germany, 201-229.

[4] Engle, R.F. and Kroner, F. (1995) Multivariate Simultaneous Generalized ARCH. Econometric Theory, 11, $122-150$. 
http://dx.doi.org/10.1017/S0266466600009063

[5] Morgan, J.P. (1996) Riskmetrics Technical Document. 4th Edition, Location, New York. http://dx.doi.org/10.1007/s10260-015-0332-9

[6] Bollerslev, T. (1990) Modelling the Coherence in Short-Run Nominal Exchange Rates: A Multivariate Generalized ARCH Approach. Review of Economics and Statistics, 72, 489-505. http://dx.doi.org/10.2307/2109358

[7] Engle, R.F. and Gonzalez-Rivera, G. (1991) Semiparametric ARCH Models. Journal of Business and Economic Statistics, 9, 345-59. http://dx.doi.org/10.1080/07350015.1991.10509863

[8] Alexander, C.O. (2002) Principal Component Models for Generating Large GARCH Covariance Matrices. Economic Notes, 31, 337-359. http://dx.doi.org/10.1111/1468-0300.00089

[9] van der Weide, R. (2002) GO-GARCH: A Multivariate Generalized Orthogonal GARCH Model. Journal of Applied Econometrics, 17, 549-564. http://dx.doi.org/10.1002/jae.688

[10] Vrontos, I., Dellaportas, P. and Politis, V. (2003) A Full-Factor Multivariate GARCH Model. Econometrics Journal, 6, 311-333. http://dx.doi.org/10.1111/1368-423X.t01-1-00111

[11] Fan, J., Wang, M. and Yao, Q. (2008) Modelling Multivariate Volatilities via Conditionally Uncorrelated Components. Journal of Royal Statistical Society B, 70, 679-702. http://dx.doi.org/10.1111/j.1467-9868.2008.00654.x

[12] Engle, R.F. (2002) Dynamic Conditional Correlation: A Simple Class of Multivariate GARCH Models. Journal of Business and Economic Statistics, 20, 339-350. http://dx.doi.org/10.1198/073500102288618487

[13] Tse, Y. and Tsui, A. (2002) A Multivariate GARCH Model with Time-Varying Correlations. Journal of Business and Economic Statistics, 20, 351-362.

[14] Cappiello, L., Engle, R.F. and Sheppard, K. (2006) Asymmetric Dynamics in the Correlations of Global Equity and Bond Returns. Journal of Financial Econometrics, 4, 537-572. http://dx.doi.org/10.1093/jjfinec/nbl005

[15] Engle, R.F. and Kelly, B. (2008) Dynamic Equicorrelation. Journal of Business and Economic Statistics, 30, $212-228$. http://dx.doi.org/10.1080/07350015.2011.652048

[16] Engle, R.F. (2009) High Dimension Dynamic Correlations. In: Castle, J. and Shephard, N., Eds., The Methodology and Practice of Econometrics: A Festschrift in Honour of David F. Hendry, Oxford University Press, Oxford. http://dx.doi.org/10.1093/acprof:oso/9780199237197.003.0005

[17] Pakel, C., Shephard, D., Sheppard, K. and Engle, R.F. (2014) Fitting Vast Dimensional Time-Varying Covariance Models. New York University, Mimeo, New York.

[18] Morana, C. (2015) The US\$/Exchange Rate: Structural Modeling and Forecasting during the Recent Financial Crisis. Università di Milano-Bicocca, Mimeo, Department of Economics, Management and Statistics, University of Milan Bicocca, Working Paper No. 321.

[19] Ledoit, O. and Wolf, M. (2004) Honey, I Shrunk the Sample Covariance Matrix. Journal of Portfolio Management, 30, 110-119. http://dx.doi.org/10.3905/jpm.2004.110

[20] Ledoit, O. and Wolf, M. (2012) Nonlinear Shrinkage Estimation of Large-Dimensional Covariance Matrices. The Annals of Statistics, 40, 1024-1060. http://dx.doi.org/10.1214/12-AOS989 